\title{
Un pájaro azul en Costa Rica: la función de la traducción de L'Oiseau bleu en la Costa Rica de $1912^{1}$
}

\author{
Francisco Javier Vargas Gómez ${ }^{2}$ \\ Universidad Nacional, Costa Rica
}

\section{RESUMEN}

El estudio procura desvelar la función de El pájaro azul (traducción de Roberto Brenes Mesén) dentro del contexto histórico en que surge. Tras describir y analizar las especificidades ideológicas, sociales y literarias de contexto, individuos y textos involucrados, se concluye que la traducción pudo haber funcionado como un instrumento para promover cambios ideológicos, sociales y estéticos dentro de la sociedad costarricense de principios del siglo veinte.

\begin{abstract}
This study seeks to describe the role of El pájaro azul (translated by Roberto Brenes Mesén) when it first appeared in Costa Rica in 1912. A description is provided of the ideological, social and literary features present in the context, and of the agents and texts involved in the production of El pájaro azul. The analysis of these features makes it possible to state that the text is likely to have been an
\end{abstract}

${ }^{1}$ Este artículo se deriva de los resultados de un trabajo final de investigación realizado para el curso «La historia de la traducción y de la teoría de la traducción hasta el siglo XIX», impartido por el Dr. Miguel Ángel Vega Cernuda, como parte del Programa de Doctorado en Traducción e Interpretación de la Universidad de Alicante, España, durante el curso lectivo 2007-2008. Dicho trabajo aparece en su totalidad en la página del grupo investigador HISTRAD (<http://web.ua.es/es/histrad/documentacion/endo-traduccion/endo-traduccionpor-naciones.html\#Costa $>$ ), dirigido por Vega Cernuda.

${ }^{2}$ Correo electrónico: saussurre2@gmail.com

$$
\text { LETRAS } 45 \text { (2009), ISSN 1409-424X }
$$


instrument used to promote ideological, social and aesthetic changes within the $20^{\text {th }}$ century Costa Rican society.

Palabras clave: modernismo, modernismo costarricense, traducción literaria, literatura e ideología.

Keywords: modernism. Costa Rican modernism, literary translation, literature and ideology.

\section{Introducción}

El presente trabajo se ubica dentro del ámbito de la Historia de la traducción y sigue una línea investigativa que busca contrastar factores histórico-sociales con la traducción y así determinar la probable función que la última cumple dentro de los primeros.

Se somete a estudio, en primer lugar, el contexto que supuso el modernismo literario en Costa Rica (como movimiento epocal ${ }^{3}$ ) de principios del siglo $\mathrm{XX}$, con sus características de tipo social, ideológico y literario; en segundo lugar, la función que cumplió dentro de tal contexto la versión al castellano de la obra de Maurice Maeterlinck, L'Oiseau bleu ${ }^{4}$, traducida por Roberto Brenes Mesén, editada por Joaquín García Monge y publicada en Costa Rica en 1912, con el título El pájaro azul 5 .

${ }^{3}$ En cuanto al carácter epocal del modernismo, sobre todo en contraposición a la visualización de tal movimiento como escuela estética o literaria, vid.: Ana Cecilia Barrantes de Bermejo, Buscando las raíces del modernismo en Costa Rica. Cinco Acercamientos (Heredia: EUNA, 1997) 9, 13, 14, 57, 58, 128-129, 162; Federico de Onís, Antología de la poesía española e hispanoamericana, 1882-1932 (Madrid: Publicaciones de la Revista de Filología Española, 1934) 149.

${ }^{4}$ Maurice Maeterlinck. 1906. L'Oiseau bleu. Ebooks Libres et Gratuits. 10 de julio 2008. <www.ebooksgratuits.com/pdf/maeterlinck_maurice_oiseau_bleu.pdf>.

${ }^{5}$ Roberto Brenes Mesén, trad. El pájaro azul «de» Maurice Maeterlinck. Cultura. Selección de buenos autores antiguos y modernos I (1916), 4: 1-77, 5: 81-173. 202 
Será pues el contexto modernista costarricense, hacia 1912, en conjunto con aquellos que promovieron la producción de la traducción mencionada lo que constituyó el objeto de estudio sobre el que versa este artículo, todo en relación a la función que cumple El pájaro azul como traducción dentro del momento y del lugar en que surgió.

\section{¿Por qué?}

Si bien El pájaro azul es un hecho conocido, no lo son tanto las razones que justifican su aparición, como traducción, en aquel momento y lugar. Partiendo de que traductor y traducción tienen un objetivo por cumplir en el contexto en que surgen, el problema principal que se aborda en estas páginas es el siguiente: ¿cuál fue la función que cumplió la traducción de L'Oiseau Blue que publicaron Roberto Brenes Mesén y Joaquín García Monge en 1912, dentro del contexto histórico en que surge y que justificaría su producción?

Tal interrogante implicó indagar sobre las características del contexto, de los participantes de la traducción, de la traducción y del «original», así como sobre la forma en que se relacionan todos estos factores. En un sentido amplio y en términos traductológicos, este artículo busca arrojar luz sobre cómo interactúan los contextos en que se dan las traducciones, los participantes del proceso y las traducciones en sí para que estas últimas surjan en momentos históricos específicos. En un ámbito más específico, la investigación es un paso más en un esfuerzo por desvelar las especificidades de la historia de la traducción literaria dentro de Costa Rica en un momento dado.

\section{¿Qué aspectos del problema se tratan?}

En vista de que lo que interesa es dilucidar la función de $E l$ pájaro azul como traducción en un contexto dado, se analiza concretamente el cuál relativo a la función, pero de igual importancia será dilucidar el porqué de tal función. Estos dos aspectos implican conocer a profundidad quiénes estuvieron involucrados, así como cuándo, cómo y dónde, aspectos todos que permitirán establecer 
relaciones entre sí mismos que servirán de base para desvelar los dos primeros.

\section{¿Desde qué perspectiva?}

Se parte de que, en consonancia con las orientaciones modernistas de aquellos que propulsaron su producción, el objeto de estudio ( $E l$ pájaro azul) cumplió una función subversiva y transformadora, cuyo propósito era promover cambios a lo interno del contexto en que se dio, sobre todo en los ámbitos ideológico y social. En concordancia con lo expuesto, mi objetivo general será determinar la función que la versión al castellano de L'Oiseau bleu (El pájaro $a z u l)$ tuvo en relación al contexto histórico, ideológico, social y literario de recepción en que surgió, a partir del análisis de diversos factores extratextuales y textuales.

En cuanto a la metodología, se optó por un análisis sociológico-cultural (Lépinette ${ }^{6}$ ), llevado a cabo en tres etapas (Pym ${ }^{7}$; López Alcalá ${ }^{8}$ ): una primera fase de recopilación y descripción de datos de carácter histórico vinculados a esferas contextuales, extratextuales y textuales en relación al objeto de estudio; una segunda fase de crítica o análisis de los datos recopilados destinada al establecimiento de relaciones entre los mismos y de generalizaciones de comportamiento a partir de tales relaciones; y una última fase en la cual se establecen, por una parte, la función del texto objeto de estudio dentro de su contexto y, por otra, las razones o condicionantes que lo llevaron a desempeñar tal función en un momento y lugar específicos.

6 Brigitte Lépinette, «La historia de la traducción-metodología. Apuntes bibliográficos», LynX Documentos de Trabajo, (1997) 14: 1-24.

${ }^{7}$ Anthony Pym, Method in Translation History (Manchester: St. Jerome, 1993).

${ }^{8}$ Samuel López Alcalá, La historia, la traducción y el control del pasado (Madrid: Universidad Pontificia Comillas, 2001). 


\section{Consideraciones teóricas}

El enfoque sociológico y cultural de este trabajo, así como su hincapié en aspectos ideológicos, sociales y literarios propios de los diversos factores que rodean al texto objeto de estudio parecen estar en armonía con la visión de las teorías polisistémicas de la traducción. Se sabe que las teorías polisistémicas suelen abordar la traducción como una práctica concebida dentro de un contexto cultural e histórico específico, y las traducciones como el producto de una sociedad específica; por tanto, condicionadas en mayor o menor medida a ella. Tales presupuestos corresponden a algunas de las formulaciones de Gideon Toury ${ }^{9}$ y André Lefevere ${ }^{10}$, basados a su vez en los que se resumen a continuación:

1. La cultura da forma a los textos (literarios) por medio de la reescritura, y a la vez, por medio de los mecanismos que controlan la escritura y reescritura; perpetúa ciertos rasgos [y productos] propios de sí misma y excluye o margina otros, pero siempre con espacio para la disensión ${ }^{11}$.

2. La crítica literaria es un intento de orientar el desarrollo de una literatura en ciertas direcciones, que coincidirán con las dictadas por la ideología y la poética dominantes del momento.

3. La literatura o el sistema literario es parte de un «(super)system» denominado sociedad, la cual, con el conjunto de todos los otros sistemas dentro de ella, constituye el «environment» del sistema

${ }^{9}$ Gideon Toury, Descriptive Translation Studies and Beyond (Ámsterdam: John Benjamins, 1995).

10 André Lefevere, «Why Waste our Time in Rewrites? The Trouble with Interpretation and the Role of Rewriting in an Alternative Paradigm» (1984), Theo Hermans (Ed.), The Manipulation of Literature: Studies in Literary Translation (Londres: Croom Helm, 1985) 215-243; André Lefevere, Traducción, reescritura y la manipulación del canon literario. Trad. $\mathrm{M}^{\mathrm{a}}$ Carmen África Vidal y Román Álvarez (Salamanca: Ediciones Colegio de España, 1997).

${ }^{11}$ Lefevere (1984) 217 y ss. 
literario; todos los sistemas en este «environment» están abiertos unos a otros ${ }^{12}$.

4. La estabilidad del sistema literario se ve amenazada por una tendencia de éste a volverse contra sí mismo y a cambiar con del tiempo.

5. Las culturas acuden a la traducción como un medio de subsanar sus carencias, sea que éstas se den como tales, o en la forma de una contraparte con la cual hacer posible una comparación ${ }^{13}$.

6. La traducción es un fenómeno regulado por los condicionantes del [o de los] sistema/s que intervienen en — todas las etapas de - su producción $^{14}$.

La traducción se concibe aquí como una actividad que toma lugar dentro de un sistema de sistemas y depende de un conjunto de condicionantes de orden histórico-ideológico-poético-social propio del contexto de recepción, condicionantes a los cuales también está supeditada la función ratificadora, subversiva, modificadora, transformadora, inventiva y/o manipuladora que sus productos (vistos como armas para impulsar o refrenar cambios ideológicos, sociales y poéticos) pueden tener en el contexto de recepción en un momento y lugar dados.

Tales postulados se complementan en este caso, en cuanto al modernismo costarricense, con los siguientes presupuestos de carácter contextual extraídos de la obra de Ana Cecilia Barrantes ${ }^{15}$ :

1. Dentro del contexto histórico en que surge El pájaro azul conviven al menos dos corrientes ideológicas en pugna: la positivista, vista

${ }^{12}$ Lefevere (1984) 216.

13 Toury, 27.

${ }^{14}$ Lefevere (1984) 234-235. Para él, la traducción, como forma de rescritura, está regulada por cinco condicionantes propios des sistema de recepción: la ideología, la poética, el universo del discurso, las especificidades del lenguaje y el original. El sistema también cuenta con dos mecanismos de control (mecenazgo y profesionales) que actúan sobre el componente ideológico y poético de las rescrituras (traducciones en este caso) haciendo que estas se ajusten a la ideología y poética dominantes dentro del sistema meta o contexto de recepción.

${ }^{15}$ Barrantes, 1997. 
como ideología dominante (y en aparente decadencia), y la modernista, vista como movimiento subversivo o contestatario.

2. El modernismo de Roberto Brenes Mesén es un movimiento que, si bien implica lo literario y estético, también abarca lo social, ideológico, cultural y pedagógico incluso de manera más contundente.

Así, la función que cumple El pájaro azul en el contexto costarricense de 1912 responde a los intereses sobre todo sociales, ideológicos y pedagógicos del proyecto modernista de Roberto Brenes Mesén (el traductor) y de Joaquín García Monge (el editor); y en menor medida, a intereses de tipo estético o literario. De ello se infiere que el texto fue utilizado como un arma propulsora de cambios en la ideología dominante del momento así como en el aspecto social (como ese espacio de disensión lefevereana al que se hace referencia en el presupuesto 3).

\section{Consideraciones metodológicas}

Según Brigitte Lépinette (1997), uno de los modelos por seguir al realizar el tipo de estudio descrito en estas páginas se denomina «modelo sociológico-cultural», el cual involucra el análisis de datos de carácter contextual y extratextual, así como los relativos a la recepción del texto producido en la lengua meta. Debido al enfoque teórico seleccionado y al objetivo prefijado, al realizar el estudio se hicieron ciertas modificaciones al método descrito por Lépinette, siendo el propio objetivo general la principal.

Por otro lado, se siguieron las tres etapas descritas por Anthony Pym (1993) con respecto al proceso de investigación histórica en traducción (etapas arqueológica, crítica y explicativa ${ }^{16}$ ), también en armonía con el carácter descriptivista de las propuestas teóricas contempladas en este estudio y con los objetivos indicados: determinar las características del entorno social, ideológico y literario en que surge la traducción, describir las características ideológico-

${ }^{16}$ Cf. López Alcalá, 110. 
sociales referentes a los involucrados en la producción de El pájaro $a z u l$, acotar las características y filiaciones ideológicas, sociales y literarias del objeto de estudio, establecer relaciones en cuanto a la afinidad o discordancia social, ideológica y literaria entre los factores antes delimitados a fin de extraer generalizaciones en cuanto a los aspectos sociales, ideológicos y literarios, y, finalmente, delinear relaciones entre las generalizaciones obtenidas y las especificidades socio-ideológico-literarias presentes en El pájaro azul de Brenes Mesén.

Se acudió a varias fuentes sobre todo de carácter bibliográfico y electrónico; entre ellas el estudio mencionado de Ana Cecilia Barrantes, en el cual se hace una descripción detallada tanto de las especificidades del contexto modernista costarricense de principios del siglo pasado como de sus principales figuras. Fundamental también, esta vez en cuanto al campo estético del modernismo en Costa Rica, es la tesis doctoral de Carlos Francisco Monge ${ }^{17}$, en la que se analizan las especificidades de la estética de los modernistas costarricenses.

En cuanto al texto objeto de estudio, ha sido muy útil el artículo de Juan Pedro Sánchez Sánchez ${ }^{18}$, en el cual se sitúa la obra en la corriente modernista. Se debe mencionar también el prólogo que incluye $E l$ pájaro azul escrito originalmente por la que fuera esposa del Maeterlinck, Georgette Leblanc ${ }^{19}$, quien ofrece datos y apreciaciones muy valiosas acerca de la evolución ideológica y filosófica de Maeterlinck y de cómo se refleja en el tono y en las características de sus obras.

${ }^{17}$ Carlos Francisco Monge Meza, Códigos estéticos en la poesía de Costa Rica (tesis doctoral) (Madrid: Universidad Complutense de Madrid, 1992).

18 Juan Pedro Sánchez Sánchez, 2003. «Ética y estética en El pájaro azul de Maeterlinck». Espéculo. Revista de estudios literarios 8/24. 30 de julio de 2008. < http://www.ucm.es/info/especulo/numero24/p_azul.html >.

${ }^{19}$ Georgette Leblanc, s.f., «Maurice Maeterlinck», Prólogo a Roberto Brenes Mesén, trad. El pájaro azul «de» Maeterlinck, Maurice, Cultura. Selección de Buenos autores antiguos y modernos I (1916), 4: 1-77, 5: 81-173. 
Queda por mencionar el binomio compuesto por L'Oiseau bleu de Maeterlinck y El pájaro azul de Brenes Mesén, los cuales como texto «original» y traducción ofrecen también datos de carácter ideológico, social y estético desde los cuales se puede inducir, en relación a los datos extraídos de las fuentes ya referidas, la función del texto objeto de estudio.

En cuanto a la metodología de análisis, se dispuso que en un primer momento se presentaran los datos obtenidos clasificados en tres esferas diferentes: la contextual, la extratextual y la textual. La esfera contextual se dividió a su vez en tres áreas de influencia que debían ser descritas, a saber, la ideológica, la social y la literaria. Así, la descripción se centró en las corrientes de pensamiento propias de la clase dominante así como la que permeaba entre las generaciones jóvenes, con sus repercusiones sobre la vida política y económica del país. Seguidamente se retrataron las características de la realidad social de la sociedad costarricense de principios del siglo XX. El panorama literario, sus tendencias y adeptos, se delimitó como factor último de la esfera contextual en este caso.

La esfera extratextual implicó la descripción, desde puntos de vista ideológico y social, de los participantes en la producción del texto estudiado, del medio en que se publicó y de los lectores a quienes iba dirigido. Tal descripción se centró entonces en las tendencias ideológico-sociales del traductor, Roberto Brenes Mesén, y del editor, Joaquín García Monge. Por otra parte, se delimitaron también el propósito y la filosofía detrás de la «Colección Ariel», de cual formó parte la traducción ahora analizada. Por último, se demarcaron las necesidades y preferencias ideológicas, sociales y literarias de ciertos grupos de jóvenes costarricenses de la época, los mismos que conformaban el público meta de la traducción de Brenes Mesén y de la misma «Colección Ariel».

El estudio de la esfera textual involucró la descripción de las especificidades ideológico-estéticas del texto estudiado y del autor del texto «original». Se describen entonces las características generales de la obra de Maeterlinck en su fase modernista, las cuales valen para la 
traducción estudiada en este caso y para su traductor. Por otra parte, también se detalla el tipo de discurso (moralizante e idealista) que siguen ambos textos, así como ciertos símbolos, elementos y temas propios de las tendencias modernistas presentes en las obras mencionadas.

Terminada la fase anterior, se extrajeron generalizaciones de carácter social, ideológico y literario para cada una de las esferas ya mencionadas. En un tercer paso, se establecieron relaciones y nuevas generalizaciones entre las especificidades de las tres esferas caracterizadas y los condicionantes de la traducción establecidos teóricamente, lo cual dio paso a una delimitación preliminar de la función del texto en su contexto de producción.

Posteriormente se inició al proceso de falsación de las hipótesis preliminares, mediante su contraposición a la función preliminar establecida en el paso anterior. Tal proceso permitió la generación de una máxima con la cual se intentó delimitar una forma precisa el comportamiento del objeto de estudio en un contexto dado y así responder la pregunta inicial. Finalmente se analizaron las implicaciones de los resultados obtenidos sobre el componente teórico.

\section{Análisis}

A partir de aquí, procedo a obtener una generalización que dé cuenta de la función que $E l$ pájaro azul de Brenes Mesén, como traducción y literatura extranjera, cumplió en el contexto modernista costarricense.

\section{Recapitulación: especificidades}

Ya que es a partir de las especificidades de las esferas descritas que surge la generalización a la que se ha hecho referencia, se sintetizan ahora tales especificidades. En cuanto a la esfera contextual (la Costa Rica de principios del siglo XX), desde el punto de vista ideológico se contrapone un positivismo de carácter liberal propio de 
las clases dominantes a un idealismo krausista contestatario que rechazaba el mercantilismo, el materialismo y el utilitarismo, que promulga el acercamiento a lo ético, a lo estético y a la naturaleza, y que percibía lo cultural como manifestación de la conciencia social.

Socialmente se visualiza un entorno en auge comercial y económico, cuyos beneficios eran acaparados por una oligarquía consumista integrada por personas económica y políticamente poderosas vinculadas a la agroexportación, y por una burguesía urbana conformada por profesionales e intelectuales tendientes al utilitarismo. En el otro extremo estaban las clases obrera y campesina, entre quienes se encuentra la juventud modernista y ciertos grupos de inmigrantes. Es una sociedad en la que existían altos niveles de analfabetismo a pesar del apogeo económico.

Con respecto al ámbito literario, el nacionalismo se muestra dominante y se manifiesta en un costumbrismo con dejos de realismo institucionalizado, cuya función era reflejar la realidad local en busca de una identidad nacional. Por otro lado, estaba el modernismo literario costarricense, que, al tiempo que criticaba y rechazaba la realidad inmediata y el localismo, idealizaba el mundo, buscaba la armonía, se decantaba por lo exótico y cosmopolita, y prefería lo ilusorio a lo real.

En la esfera extratextual, se tiene a un traductor partidario de un idealismo espiritual de corte modernista proyectado en su obra poética y que extiende al ámbito social por medio de su obra y de su incursión en el campo educativo, y cuyas tendencias literarias lo apartan y enfrentan al costumbrismo reinante de la época. El editor es una figura cuya ideología idealista-humanista, planteada alrededor de un ser humano y un mundo nuevos, lo hace combatir la plutocracia, el positivismo y el liberalismo de la clase dominante, que lo conduce en lo social a aspirar a una nueva sociedad más igualitaria, y que ve en la educación la solución a los problemas sociales, y en la literatura un arma para concientizar al pueblo y promover el cambio. El lector meta (jóvenes y obreros de clases más bien bajas) adopta un idealismo modernista de corte local en el plano ideológico, que lo lleva, en el 
plano social, a rechazar a la clase burguesa y oligarca dominante, y a identificarse con las clases obrera y campesina, y cuyos gustos literarios se inclinan hacia los autores modernistas sudamericanos en cuyos textos buscan la realidad dejando de lado su propio entorno.

Finalmente, el medio en que se publica la obra da cuenta de una marcada ideología modernista en tanto es una publicación que por sus contenidos se muestra ecléctica, abierta a lo extranjero y exótico, que privilegia a los autores modernistas e idealistas de diferentes latitudes, y que en lo social se idea como una herramienta para instruir al pueblo y promover la reflexión en las clases bajas rebosantes de jóvenes y obreros, así como el cambio hacia una sociedad nueva.

Dentro de la última esfera, la textual, la obra analizada revela una marcada ideología y estética modernistas, en torno a las cuales se construyen desde los temas hasta los personajes, pasando por el argumento, las ubicaciones espacial y temporal, los diálogos, los decorados y el vestuario, ente otros. Esta ideología modernista de la obra la conduce a presentar ante la audiencia o el lector una fuerte crítica al materialismo y al utilitarismo reinantes en la época, una revaloración de lo sencillo y del arte, y una lucha a favor de lo espiritual y de los ideales más altos, contraposición pues entre lo real y lo ideal, entre material y los ideales, entre lo que es y lo que debería ser. Su estética modernista, opuesta a la del naturalismo literario, se denota en la selección de temas de corte universal, en la no delimitación de tiempos y espacios específicos, en la preferencia por lo exótico y extranjero, en el diálogo entre la naturaleza y el ser humano y en la contraposición entre el mundo real y el de ensueño.

\section{Generalizaciones: los condicionantes de la traducción}

Es posible ya extraer generalizaciones (una para cada esfera) con las cuales describir la posición de los condicionantes de la traducción pertenecientes al sistema meta (ideología y poética) y de los mecanismos de control (mecenazgo y profesional) que condicionaron la producción del texto estudiado y que delimitarían su función dentro de dicho sistema. 
En cuanto al ámbito contextual, en el caso de la Costa Rica de principios de siglo pasado existió un ambiente bifurcado o dualidad de carácter ideológico, social y estético-literario, dentro del sistema de sistemas que es la sociedad; en tales circunstancias existían al menos dos ideologías y dos poéticas en pugna a las cuales podían o debían adaptarse las diferentes formas de reescritura que se produjeran en aquel momento.

Extratextualmente, a diferencia de lo que sucedía en la esfera anterior, existió uniformidad de criterios en los factores que rodearon la producción del texto estudiado. Tal uniformidad (en cuanto a las tendencias ideológicas, sociales y literarias de quienes intervinieron en dicha producción) giraba en torno al idealismo-modernista. Por ello, es posible que los mecanismos de control (mecenazgo y profesional) que regularon la aparición del texto estudiado dentro del sistema meta (Costa Rica) favorecieran la producción y diseminación de productos que manifestasen una ideología y estética modernistas. Aunque sin formar parte del grupo dominante, tales mecanismos sí contaban con los medios para producir, publicar y distribuir textos/traducciones afines a sus intereses.

Al igual que en la esfera extratextual, el texto estudiado y sus distintos componentes también se muestran amalgamados en torno a una única corriente ideológico-estética de índole modernista. No fue aquel un texto que estuviera en sintonía con los intereses ideológicos, sociales y estéticos de aquellos que eran parte de la élite dominante, sino de quienes se oponían a ella. Era un tipo de literatura, hasta cierto punto, disidente.

\section{La función del texto meta en el contexto de recepción}

Partiendo de las tres aseveraciones anteriores, y aceptando que con ellas se ha descrito la posición ideológica y poética que los condicionantes y mecanismos de control propios del sistema de recepción (Costa Rica) sostenían en torno al texto meta (El pájaro $a z u l)$, es posible ahora intentar inducir una concepción - todavía 
preliminar- de la función que la traducción cumplió dentro del contexto histórico, ideológico, social y literario en que surgió.

Como producto de un contexto histórico, ideológico, social y literario costarricense de principios del siglo Xx que ofrecía al menos dos opciones ideológicas y poéticas (una dominante y la otra disidente), y habiendo sido producido por un mecenazgo y un profesional (editor $\mathrm{y}$ traductor) en apariencia no adeptos ideológicamente a la clase dominante, sino partidarios de la oferta ideológica y poética disidente, y promotores de la aparición y utilización de determinados textos como método y herramientas para restar preeminencia a la clase, ideología y poética dominantes, el texto estudiado cumpliese la función de «arma» disidente, subversiva, trasgresora y transformadora, entre otras, utilizada a favor de los intereses ideológicos, sociales y poéticos de quienes promovieron su producción y en contra de la ideología y poética dominantes.

\section{Falsación: la función delimitada y las hipótesis preliminares}

La hipótesis de que la función del texto estudiado dentro del contexto de recepción respondió principalmente a los intereses sociales, ideológicos y pedagógicos del proyecto modernista de Roberto Brenes Mesén y de Joaquín García Monge y en menor medida a intereses de tipo estéticos y/o literarios ha sido validada por el análisis pero sólo parcialmente.

Si bien el texto estudiado está en consonancia con los intereses de carácter social, ideológico y pedagógico del modernismo de Brenes Mesén y García Monge, no responde a intereses estéticos y literarios en menor medida. En el modernismo en general y en el costarricense en particular, así como sus representantes y adeptos, se consideraba que la búsqueda del ideal estético era también la búsqueda de la armonía y de la renovación, incluidas la armonía y la renovación en el ámbito ideológico y social.

El vínculo entre el componente estético y el ideológico-social, por ejemplo, queda claro al observar la búsqueda modernista del ritmo poético. Como indica Barrantes, ese afán por encontrar tal ritmo como 
manifestación del ritmo o armonía universal les permite encontrar basamentos ideológicos con los cuales minar las propuestas positivistas y les conduce hacia las cuestiones sociales, ya que el ritmo poético era inútil si la modernidad no los elevaba a todos por igual. Así, en cuanto a la cuestión social, «en el caso costarricense, es harto difícil deslindar lo estético de su contexto social pues el modernismo invade determinantemente las cuestiones sociales al afirmarse... el deseo de renovación del pensamiento costarricense y el afán por romper los cánones seculares que aletargaban a la sociedad» ${ }^{20}$.

Por otro lado, es posible plantear que la enseñanza de lo estético por lo tanto conllevaba la enseñanza en otros ámbitos. De ahí que tampoco se pueda separar lo pedagógico de lo estético ni de lo literario. En lo cuanto a la segunda hipótesis, esta parece validada con el análisis de los datos. El pájaro azul parece haberse convertido en uno más del conjunto de instrumentos que los modernistas costarricenses pudieron haber utilizado para promover una actitud disidente y contestataria en los lectores, y así propiciar cambios dentro del sistema de recepción en diferentes ámbitos. En este caso, todas las circunstancias fueron propicias para promover tal función: 1. la existencia de un movimiento ideológico (Positivismo) y poético (Nacionalismo) opuesto al representado por el texto estudiado; 2. el que tal movimiento fuera hegemónico y adoptado por la clase dominante; 3. el que la ideología y poética idealista-modernista representadas por el texto estudiado contara con cierto peso dentro del conjunto de la sociedad a pesar de no ser dominantes; 4. el que quienes promueven su aparición tuviesen acceso a los medios para explotar el potencial subversivo y transformador que tenía la obra dentro de ese contexto; y 5. el que existiera una masa de lectores que «demandaba» ese tipo de material.

${ }^{20}$ Barrantes, 15-16. 


\section{Conclusión}

Dentro del contexto histórico que representa la Costa Rica de 1912, El pájaro azul funcionó como un medio para propagar las ideas modernistas e idealistas de quienes estaban detrás de su producción y publicación entre un sector de la población perteneciente a las clases medias y bajas que se encontraba descontento con el proceder de la clase dominante, todo con el fin último de promover cambios a nivel ideológico, social y estético-literario dentro de la sociedad en que surge como parte de un proyecto pedagógico más amplio. Como traducción y literatura extranjera, El pájaro azul debió haber sido un instrumento de disidencia y transformación ideológico, social y estético-literario.

Este carácter subversivo y modificador sería el resultado de que, en tanto producto de la reescritura, El pájaro azul estuvo ideológica y estéticamente en consonancia con las especificidades de una ideología y de una poética (modernistas) que no eran las dominantes dentro del sistema de recepción, y de que su producción estuvo en función de los intereses de un mecenazgo, un profesional y un lector meta no pertenecientes al grupo dominante de la sociedad.

Tanto la función del texto estudiado como sus aparentes causas brindan espacio para plantear algunas ideas en torno a los presupuestos teóricos que fundamentan el estudio. Para ello hay que retomar la idea de Lefevere de que los sistemas literarios, y aquí habrá que extender la afirmación a todos los demás sistemas, tienden a volverse contra sí mismos y a «mutar». Esto abriría una brecha para la disidencia a la que el mismo Lefevere hace referencia. Dentro del contexto estudiado parecen presentarse ese proceso de volverse contra sí mismo y ese espacio para la disidencia; el primero en un estado algo avanzado (no que se llegue a concretar, sino más bien parece alcanzar una especie de balance entre las dos fuerzas en pugna); el segundo lo suficientemente amplio como para que convivan diferentes realidades de poder y de autoridad que dan origen a la convivencia de dos grupos contrapuestos de condicionantes de la reescritura y de mecanismos de control, ambos con los medios suficientes como para promover la 
producción de productos afines a sus tendencias ideológicas y poéticas y para garantizar a los lectores el acceso a ellos.

Esta inestabilidad en el sistema resulta de interés en el caso de Costa Rica puesto que, si se observa el devenir literario del país luego de 1912, parece mantenerse de la misma forma durante cierto período, sin que ninguna de las dos ideologías o poéticas llegase a ser claramente dominante en lo literario, sino más bien a establecer una especie de «convivencia pacífica». Por lo demás, parece que tanto el enfoque lefevereano a la investigación en traducción («systems approach») como la concepción de la traducción delimitada en la sección introductoria de este trabajo han resultado apropiadas y funcionales en este caso.

El efecto que tuvo el texto estudiado dentro del contexto en que se le ha ubicado está aún por determinarse; de ahí el que se planteen afirmaciones solamente en torno a una función potencial del texto dentro de su contexto de recepción de acuerdo a las posibilidades que ha arrojado el análisis de las especificidades de las distintas esferas en que se dio la aparición de El pájaro azul. La confirmación de las propuestas que aquí se presentan estaría supeditada al estudio de la respuesta por parte de los diferentes sectores de la sociedad al texto estudiado (el impacto del texto), observable mediante reseñas críticas, antologías y otros metatextos, del volumen de ventas, del número de reimpresiones y del alcance general de la obra, entre otras fuentes.

Aun así, es presumible que, como pieza de un proyecto de cambio ideológico, social y literario de corte modernista, la naturaleza modernista del El pájaro azul debió de afectar en mayor o menor grado la sensibilidad ideológica, moral y estética así como las conductas de quienes llegaron a leer la obra, lo cual, es de suponer, incidiría también en mayor o menor grado en el aspecto social. Es claro que sus características le otorgaban tal potencial dentro del contexto histórico de una Costa Rica de principios del siglo XX, cuando Brenes Mesén y García Monge, así como otros modernistas costarricenses, se habían propuesto generar un cambio. 